\title{
ON THE COMPLETENESS OF HAMILTONIAN VECTOR FIELDS
}

\author{
WILLIAM B. GORDON
}

\begin{abstract}
Sufficient conditions are given for a hamiltonian vector field to be complete which involve bounds on the potential or its gradient.
\end{abstract}

1. A vector field $\xi$ on a manifold $M$ is said to be complete iff for every $m \in M$ the maximal interval of existence $\left(\omega_{-}, \omega_{+}\right)$of every solution of

$$
d x / d t=\xi, \quad x(0)=m,
$$

is given by $\omega_{ \pm}= \pm \infty$, cf. [1]. Hence if $\xi$ is of class $C^{1}$, so that solutions are unique, to say that $\xi$ is complete means that $\xi$ generates a 1parameter group or (global) flow on $M$. Let $\left(t_{-}, t_{+}\right)$be a bounded open neighborhood of 0 in which a solution $x=x(t)$ to (1) is defined. It is well known that $\omega_{ \pm}= \pm \infty$ iff $x(t)$ remains in a compact set as $t$ varies over any such neighborhood, [2]. A device which assures this property is provided by the following simple lemma.

Lemma. Let $\xi$ be a $C^{0}$ vector field on a manifold $M$ of class $C^{1}$. Then $\xi$ is complete if there exist a $C^{1}$ function $E$, a proper $C^{0}$ function $f$, and constants $\alpha, \beta$ such that for all $m \in M$

(i) $|\xi E(m)| \leqq \alpha|E(m)|$,

(ii) $|f(m)| \leqq \beta|E(m)|$.

(Recall that $f$ proper means $f^{-1}$ (compact) $=$ compact.)

Proof. From basic definitions $\xi E(m)=d E(x(t)) /\left.d t\right|_{t=0}$. Hence from Gronwall's inequality, or otherwise, it follows that $|E(x(t))|$ $\leqq|E(x(0))| e^{\alpha|t|}, \omega_{-}<t<\omega_{+}$so that $|f(x(t))| \leqq \beta|E(x(0))| e^{\alpha|t|}$. Since $f$ is proper, this means that $x(t)$ remains in a compact set as $t$ varies over a bounded neighborhood of 0 (for which a solution is defined).

2. To apply this lemma to the case $\xi=$ hamiltonian vector field, let $(M, g)$ be a riemannian manifold of class $C^{1}$ with metric tensor $g$, and let $(q, p)$ denote local coordinates on $T^{*}(M)$. Every $C^{1}$ "potential" $V$ on $M$ gives rise to a hamiltonian $H=T+V=$ kinetic energy + potential $=\frac{1}{2} \sum g^{i j} p_{i} p_{j}+V(q)$ whose corresponding hamiltonian vector

Received by the editors November 1, 1969.

AMS 1969 subject classifications. Primary 3465; Secondary 3404.

Key words and phrases. Complete vector field, hamiltonian vector field. 
field $\xi$ on $T^{*}(M)$ is given by $\xi=\sum\left\{\left(\partial H / \partial p_{i}\right) \partial / \partial q_{i}-\left(\partial H / \partial q_{i}\right) \partial / \partial p_{i}\right\}$. We now prove the following

THEOREM. Let $(M, g), \xi, V$ be as above. Then $\xi$ is complete if any of the following are true.

(i) $V$ is proper and bounded below, say $V \geqq 0$.

(ii) $(M, g)$ is complete (in the riemannian sense) and $V \geqq 0$.

(iii) $(M, g)$ is complete and $\|\nabla V\|$ is bounded.

(iv) $(M, g)$ is complete and $\|\nabla V\| \leqq$ constant $\cdot\|w\|$ where $m \rightarrow w(m)$ is an isometric embedding of $M$ into euclidean space.

Proof. For (i) apply the lemma with $f=E=H$. Since $V$ is a proper function on $M$, and $V \geqq 0$, it follows that $H$ is proper on $T^{*}(M)$. But $\xi H=0$, so that the hypotheses of the lemma are satisfied. For the remainder of the theorem, let $q \rightarrow w(q)$ be an isometric embedding of $M$ into euclidean space $R^{n}$. (The existence of such embeddings is given by a theorem of Nash [3].) Since $(M, g)$ is complete as a riemannian manifold, $M$ is complete with respect to the metric induced by $g$. Therefore $M$ is a closed submanifold of $R^{n}$. It follows that $r^{2}(m)=\|w(m)\|^{2}$ is a proper function on $M$. Also we have

$$
\left|\xi r^{2}\right|=2\left|\left\langle w, \sum p_{i} w^{i}\right\rangle\right| \leqq 2\|w\| \cdot\left\|\sum p_{i} w^{i}\right\| \leqq 2\|w\| \cdot(2 T)^{1 / 2}
$$

where $\langle$,$\rangle is the standard inner product on R^{n}, w_{i}=\partial w / \partial q_{i}$ and $w^{i}=\sum g^{i j} w_{j}$, so that $g_{i j}=\left\langle w_{i}, w_{j}\right\rangle$ and $g^{i j}=\left\langle w^{i}, w^{j}\right\rangle$. To prove (ii), set $E=H+r^{2}$. Then it is easy to show that $|\xi E / E| \leqq 2$. To obtain a function $f$ satisfying the hypotheses of the lemma, set $f=r^{2}$ (or $f=E$ ). To prove (iii) and (iv) let $f=E=T+\frac{1}{2} r^{2}$. A direct calculation shows that $|\xi T|=\left|\sum g^{i j} p_{i} \partial V / \partial q_{i}\right| \leqq(2 T)^{1 / 2} \cdot\|\nabla V\|$ so that $|\xi E / E|$ $\leqq(2 T)^{1 / 2}\|w\|(1+\|\nabla V\| /\|w\|) /\left(T+\frac{1}{2}\|w\|^{2}\right)$. The proof of (iv) is now immediate. To prove (iii) we need only choose an embedding for which $\|w(m)\|$ is bounded below by a positive number.

Note that (i) has as a consequence the well-known fact that every hamiltonian vector field attached to (the cotangent bundle of) a compact manifold is complete, this being a generalization of the fact that every compact riemannian manifold is complete in the riemannian sense, (the case $V=0$ ).

Finally, we remark that the lemma and the theorem can easily be extended to the nonautonomous case $\xi=\xi(m, t)$ by the usual device: one considers the vector field $\hat{\xi}=\hat{\xi}+\partial / \partial t$ on $M \times R$ (in the lemma) and $T^{*}(M) \times R$ (in the theorem) to obtain sufficient conditions for completeness. For example, parts (iii) and (iv) of the theorem remain true if the potential is time dependent; to see this replace the function $E$ in the argument by $\hat{E}=E+t^{2}$. Then $\hat{E}$ is a proper function 
on $T^{*}(M) \times R$ and $|\hat{\xi} \hat{E} / \hat{E}| \leqq$ constant. On the other hand, generalizations of parts (i) and (ii) apparently require boundedness conditions on $\partial V / \partial t$.

\section{BIBLIOGRAPHY}

1. R. Abraham, Foundations of mechanics, Benjamin, New York, 1967. MR 36 $\# 3527$.

2. P. Hartman, Ordinary differential equations, Wiley, New York, 1964. MR 30 $\# 1270$.

3. J. Nash, The imbedding problem for Riemannian manifolds, Ann. of Math. (2) 63 (1956), 20-63. MR 17, 782.

Naval Research Laboratory, Code 7840, Washington, D. C. 20390 